\title{
Concentración sérica de aminotransferasas en cuyes (Cavia porcellus) alimentados con dietas basadas en pisonay (Erythrina sp)
}

\section{Serum concentration of aminotranferases in guinea pigs (Cavia porcellus) fed diets based on pisonay (Erythrina sp)}

\author{
Yony Ramirez-Borda ${ }^{1}$, Ludwing A. Cárdenas-Villanueva ${ }^{1,2}$, \\ Víctor A. Ramos-De la Riva ${ }^{1}$, Oscar E. Gómez-Quispe ${ }^{1}$
}

\section{Resumen}

El experimento se llevó a cabo en Tamburco, provincia de Abancay, Perú, con el objetivo de determinar el efecto de dietas basadas en pisonay (Erythrina sp) sobre la concentración de taninos y alcaloides totales, la concentración sérica de las aminotransferasas, las lesiones en el hígado y la relación hígado/peso vivo de los cuyes. Se utilizaron hojas y peciolos de pisonay con cuatro meses de rebrote y alfalfa fresca (Medicago sativa) con 28 días de rebrote en la dieta de 24 cuyes machos mejorados, distribuidos en tres tratamientos: dieta A100: 100\% alfalfa (80 g), dieta A50P50: 50\% alfalfa (40 g) más $50 \%$ pisonay $(40 \mathrm{~g})$ y dieta $\mathrm{P} 100: 100 \%$ pisonay $(80 \mathrm{~g})$. El alimento fue suministrado dos veces al día. Los niveles séricos y la relación hígado/peso vivo fueron analizados con diseño completo al azar, el efecto de las dietas sobre las lesiones del hígado de cuyes se analizó con el test exacto de Fisher. La concentración de taninos en el pisonay (14.0 g/kg MS) fue superior a los hallados en alfalfa $(2.2 \mathrm{~g} / \mathrm{kg} \mathrm{MS})$. En la alfalfa no se detectaron alcaloides totales. Los niveles séricos de alanino aminotransferasa (ALT) en cuyes alimentados con A100 (39.1 $\pm 7.2 \mathrm{UI} / \mathrm{L}), \mathrm{A} 50 \mathrm{P} 50(48.8 \pm 7.1 \mathrm{UI} / \mathrm{L})$ y P100 $(62.0 \pm 8.4 \mathrm{UI} / \mathrm{L})$ mostraron diferencias significativas $(\mathrm{p}<0.05)$. La aspartato aminotransferasa (AST) en cuyes alimentados con A50P50 (71.9 $\pm 15.2 \mathrm{UI} / \mathrm{L})$ y P100 $(76.4 \pm 11.8 \mathrm{UI} / \mathrm{L})$ fueron superiores a los animales alimentados con A100 $(\mathrm{p}<0.05)$. El incremento de 50 a $100 \%$ de pisonay en la dieta de cuyes produce lesiones en el hígado como degeneración grasa y focos necróticos, además de disminuir la relación hígado/peso vivo. El pisonay como forraje fresco en 50 y $100 \%$ en la dieta de cuyes pueden afectar adversamente la salud de los animales.

Palabras clave: taninos; alcaloides; alanino aminotransferasa; aspartato aminotransferasa

\footnotetext{
${ }^{1}$ Laboratorio de Farmacología, Toxicología y Bioquímica, Universidad Nacional Micaela Bastidas de Apurimac, Perú

${ }^{2}$ E-mail: car-vet@hotmail.com
}

Recibido: 7 de noviembre de 2018

Aceptado para publicación: 4 de julio de 2019 
The experiment was carried out in Tamburco, province of Abancay, Peru with the objective of determining the effect of diets based on pisonay (Erythrina $\mathrm{sp}$ ) on the concentration of tannins and total alkaloids, the serum concentration of the aminotransferases, the lesions in the liver and the liver/liveweight ratio of the guinea pigs. Leaves and petioles of pisonay were used with four months of regrowth and fresh alfalfa (Medicago sativa) with 28 days of regrowth in the diet of 24 improved male guinea pigs, distributed in three treatments: diet A100: 100\% alfalfa (80 g), diet A50P50: 50\% alfalfa (40 g) plus 50\% pisonay ( $40 \mathrm{~g})$ and diet $\mathrm{P} 100: 100 \%$ pisonay $(80 \mathrm{~g})$. The feeds were supplied twice a day. Serum levels and liver/liveweight ratio were analysed with complete random design, the effect of diets on guinea pig liver lesions was analysed with Fisher's exact test. The concentration of tannins in the pisonay $(14.0 \mathrm{~g} / \mathrm{kg} \mathrm{DM})$ was higher than those found in alfalfa $(2.2 \mathrm{~g} / \mathrm{kg} \mathrm{DM})$. No total alkaloids were detected in the alfalfa. Serum levels of alanine aminotransferase (ALT) in guinea pigs fed A100 (39.1 $\pm 7.2 \mathrm{IU} / \mathrm{L}), \mathrm{A} 50 \mathrm{P} 50$ $(48.8 \pm 7.1 \mathrm{IU} / \mathrm{L})$ and P100 $(62.0 \pm 8.4 \mathrm{IU} / \mathrm{L})$ showed significant differences $(\mathrm{p}<0.05)$. Aspartate aminotransferase (AST) in guinea pigs fed A50P50 (71.9 $\pm 15.2 \mathrm{IU} / \mathrm{L})$ and P100 $(76.4 \pm 11.8 \mathrm{IU} / \mathrm{L})$ were higher than animals fed A100 $(\mathrm{p}<0.05)$. The increase of 50 to $100 \%$ of pisonay in the diet of guinea pigs produces lesions in the liver such as fatty degeneration and necrotic foci, in addition to decreasing the liver/liveweight ratio. The pisonay and fresh forage in 50 and $100 \%$ in the diet of guinea pigs can adversely affect the health of the animals.

Key words: tannin; alkaloids; alanine aminotransferase; aspartate aminotransferase

\section{INTRODUCCIÓN}

Las leguminosas arbóreas y arbustivas forrajeras tienen gran potencial multipropósito, se usan en cercos vivos, postes, sombra, medicina, utensilios, leña y en la alimentación de animales (Kongmanila et al., 2012; Yisehak y Janssens, 2013; Bohada et al., 2017). Sin embargo, tienen efectos citotóxicos, carcinogénicos, hepatotóxicos, nefrotóxicos y genotóxicos (Zhu et al., 1992; Araújo-Júnior et al., 2012). Así mismo, se ha observado disminución del consumo voluntario en el ganado (Meza et al., 2012, 2014b; Santos et al., 2014) y, por consiguiente, menor ganancia de peso vivo (Sánchez et al., 2012; Guevara et al., 2013; Meza et al., 2014a) e incluso problemas de salud. Al respecto, Apráez et al. (2013) indicaron que la elevada presencia de compuestos fenólicos en la alimentación con especies arbustivas había provocado una mortalidad de cerca del $25 \%$ en cuyes.

Ensayos cualitativos realizados en el follaje de leguminosas arbóreas y follaje de arbustos forrajeros indicaron la existencia de metabolitos secundarios como compuestos fenólicos, taninos, saponinas, alcaloides, fitatos y oxalatos, los que podrían tener un efecto negativo en el valor nutritivo del forraje (Galindo et al., 2014; Apráez et al., 2017; Milián et al., 2017). Varios extractos del género Erythrina han mostrado mayor frecuencia de alcaloides (38\%), flavonoides (38\%) y proteoides (9\%) (Pino et al., 2004). En otros estudios, E. mysorensis presentó moderada presencia de taninos, compuestos fenólicos y alcaloides (Rodríguez et al., 2004), E. berteroana solo taninos (Pedraza et al., 2007) y E. variegata una presencia baja de taninos y alta de alcaloides (Alvear et al., 2013). Asimismo, en ensayos cuantitativos 
realizados en hojas y tallos finos de arbóreas forrajeras cosechadas a los 120 días de rebrote, la concentración de taninos totales fue 1.1 a $54.2 \mathrm{~g} / \mathrm{kg}$ MS (García et al., 2008; Ogunbosoye et al., 2015).

Es conocida la utilización de follajes arbustivos y arbóreos como forraje verde o harina de hojas para la dieta de los animales. En la evaluación post mortem de conejos alimentados con Leucaena leucocephala se encontró hígado frágil e incluso congestionado con manchas necróticas, que indicaría una condición de insuficiencia orgánica múltiple como efecto de la toxicidad de taninos (Adekojo et al., 2014). En pollos, con dietas conteniendo niveles superiores a $15 \%$ de Moringa oleífera se observó disminución de los niveles séricos de alanino aminotransferasa (ALT) y aspartato aminotransferasa (AST), lo que implicaría los efectos maximizados de los factores tóxicos (Tijani et al., 2016). La ALT y AST han sido utilizadas como indicadores sensibles de citólisis o daño celular hepático, así como marcadores de daño hepatocelular (Lawrence y Steiner, 2017).

En los valles interandinos del Perú, los criadores utilizan hojas y peciolos del pisonay (Erythrina $\mathrm{sp}$ ) en la alimentación de animales rumiantes y no rumiantes, sin tomar en cuenta las consideraciones de toxicidad. Por estas razones, esta investigación tuvo como propósito determinar los niveles de taninos y alcaloides totales en la alfalfa (Medicago sativa) y pisonay, el efecto del consumo de estos forrajes sobre la concentración sérica de aminotransferasas, las lesiones patológicas en el hígado y la relación hígado/peso vivo de los cuyes.

\section{Materiales y Métodos}

El trabajo experimental fue realizado en una granja de cuyes ubicada en el sector de Moccospampa, distrito de Tamburco, provincia de Abancay, departamento de Apurímac,
Perú. La zona se encuentra a una altitud de $2880 \mathrm{~m}$, con temperatura ambiental entre 11.7 y $23.8{ }^{\circ} \mathrm{C}$, humedad relativa de $62.7 \%$ y precipitación pluvial media anual de $596 \mathrm{~mm}$.

El forraje estuvo constituido por hojas y peciolos del pisonay (Erythrina $\mathrm{sp}$ ) con un crecimiento vegetativo de 120 días de rebrote y de alfalfa (Medicago sativa) fresca de 28 días. Estos forrajes fueron cosechados en los alrededores del sitio experimental a inicios de la época seca (abril y mayo de 2016).

Se utilizaron 24 cuyes machos mejorados, aparentemente sanos, de tres meses. Los animales fueron pesados al inicio de la fase experimental ( $836 \pm 87 \mathrm{~g}$ ) en una balanza Adventurer Pro AV2101 Ohaus $( \pm 0.02 \mathrm{~g})$. Los cuyes se distribuyeron al azar en tres tratamientos. Los cuyes se criaron en pozas de adobe $(1.2 \times 0.8 \times 0.7 \mathrm{~m})$ con sus respectivos bebederos. Previa a la experimentación, los animales tuvieron un periodo de acostumbramiento a la dieta de siete días. Basado en los valores nutricionales del pisonay reportados por Cárdenas et al. (2013, 2016a,b) y Choque et al. (2018), los tratamientos tuvieron los siguientes porcentajes de forraje: A100: $100 \%$ de alfalfa ( $80 \mathrm{~g})$, A50P50: $50 \%$ de alfalfa ( $40 \mathrm{~g}$ ) y $50 \%$ de pisonay ( 40 g), P100: $100 \%$ de pisonay ( $80 \mathrm{~g}$ ). La dieta fue suministrada durante 21 días (etapa experimental), dos veces al día, previo pesaje de los forrajes (Adventurer OHAUS). El agua estuvo disponible a voluntad.

Las muestras de alfalfa y pisonay se secaron a $55^{\circ} \mathrm{C}$ por 72 horas. Se determinó la concentración de taninos totales utilizando un espectrofotómetro Lambda $25 \mathrm{UV} / \mathrm{Vis}$ Perkin Elmer con referencia al acido tánico (Velásquez, 2004) y alcaloides totales por volumetría - microbureta - con referencia a eritrinanos (AOAC, 1990). Los cuyes fueron beneficiados al término de la etapa experimental previa insensibilización por dislocación de las vértebras cervicales (JuradoGámez et al., 2016) y las muestras de sangre fueron colectadas de la vena yugular (Pilny, 2008). Las muestras de sangre (libre de 
hemolisis) fueron recolectados en tubos sin anticoagulante para la obtención del suero por centrifugación (2-16P Sigma) a $1398 \times g$ durante 10 minutos. El suero resultante fue guardado en congelación a $-20^{\circ} \mathrm{C}$. Se determinaron las concentraciones séricas de alanina aminotransferasa (ALT) y aspartato aminotransferasa (AST) en un espectrofotómetro $6540 \mathrm{UV} / \mathrm{Vis}$ Jenway con kits comerciales (Valtek Diagnostics).

Terminado el experimento se realizó la necropsia de los cuyes (Astaiza-Martínez et al., 2013), se describieron las posibles alteraciones macroscópicas presentes en el hígado, y se determinó su peso (Adventurer OHAUS) para el cálculo de la relación hígado/peso vivo.

Los valores de taninos y alcaloides en alfalfa y pisonay fueron analizados con estadística descriptiva, la respuesta de las dietas en las concentraciones séricas de ALT, AST y la relación hígado/peso vivo se analizó con un diseño completamente al azar, y la diferencia de sus promedios con la prueba de Tukey. Asimismo, el efecto de las dietas sobre las lesiones del hígado de cuyes se analizó con el test exacto de Fisher, utilizando un nivel de confianza del $95 \%$ para todos los casos.

\section{Resultados y Discusión}

Los valores de taninos y alcaloides totales fueron mayores en el pisonay que en la alfalfa. Asimismo, los alcaloides totales en la alfalfa fueron imperceptibles (Cuadro 1).

La concentración de $2.2 \mathrm{~g} / \mathrm{kg}$ de MS de alfalfa fue similar al valor de $0.125 \mathrm{~g} / \mathrm{kg}$ de MS reportado por Barry y McNabb (1999), mientras que Coblentz y Grabber (2013) no detectaron taninos en el ensilado de alfalfa y valores de $1.62 \%$ (equivalente a $16 \mathrm{~g} / \mathrm{kg} \mathrm{MS}$ ) en el heno de alfalfa. En el caso de pisonay se disponen de escasos estudios. Bhat et al. (2013) indican que los forrajes que contienen niveles bajos a moderados de taninos $(<5 \%$ de MS) pueden emplearse como alimento de forma segura para los animales; en forma similar, Mohamed et al. (2015) indican que estos forrajes representan una fuente efectiva de proteína disponible, a diferencia de aquellas sin taninos. García et al. (2009) encontró 3.5\% (35 g/kg MS) en leguminosas arbóreas y $2.4 \%$ ( $24 \mathrm{~g} / \mathrm{kg} \mathrm{MS})$ en no leguminosas, las cuales tienen efecto negativo en la digestión de la MS.

El pisonay por su bajo contenido de taninos puede ser considerado como un forraje promisorio al ser comparado con otros arbustos forrajeros (Reddy y Elanchezhian, 2008; Santacoloma y Granados, 2012; Ojeda et al., 2012; Brown et al., 2016), y considerado para la suplementación al no tener efectos negativos (Mashamaite et al., 2009). Sin embargo, en el presente trabajo se han observado valores elevados de alcaloides totales $(17.8 \mathrm{~g} / \mathrm{kg} \mathrm{MS})$, que pueden ocasionar toxicidad en el metabolismo digestivo de los animales que la consumen. Las concentraciones de alcaloides son bajas en leguminosas arbóreas (0.04-0.51\%) (García y Medina, 2006) y en no leguminosas (0.07-0.10\%) (García et al., 2008). Las concentraciones de alcaloides menores a $0.32 \pm 0.03 \%$ han sido considerados dentro del rango seguro (Cheema et al., 2011) y valores de $1.24 \pm 0.14 \%$ no ocasionarían efectos adversos en monogástricos (Alikwe y Omotosho, 2013).

El suministro de alimentos en base a pisonay en las proporciones de A50P50 y P100 de la dieta de cuyes mostró altas concentraciones de ALT y AST comparado con la dieta solo de alfalfa. Estos valores sobrepasan al rango máximo propuesto por Kitagaki et al. (2005) quienes reportan valores para ALT de 25-40 UI/L y AST de 45-68 UI/L. El incremento de las concentraciones séricas de ALT y AST indican disfunciones hepáticas (Ticona, 2011; Ruíz-Rodríguez, 2013). Sin embargo, la inclusión de harina de Erythrina sp en bajas proporciones (7-28\%) en la alimentación de cuyes no evidenciaron cambios 
Cuadro 1. Nivel de taninos y alcaloides totales ( $\mathrm{g} / \mathrm{kg}$ de MS) en alfalfa (Medicago sativa) y pisonay (Erythrina sp)

\begin{tabular}{lcccc}
\hline \multirow{2}{*}{ Forrajes } & \multicolumn{2}{c}{ Taninos totales } & \multicolumn{2}{c}{ Alcaloides totales } \\
\cline { 2 - 5 } & Media $\pm \mathrm{DE}$ & $\mathrm{CV}(\%)$ & Media $\pm \mathrm{DE}$ & $\mathrm{CV}(\%)$ \\
\hline Alfalfa & $2.2 \pm 0.2$ & 9.9 & $\mathrm{ND}$ & $\mathrm{ND}$ \\
Pisonay & $14.0 \pm 1.8$ & 12.7 & $17.8 \pm 2.3$ & 12.9 \\
\hline
\end{tabular}

DE: Desviación estándar; CV: Coeficiente de variabilidad; ND: No detectado
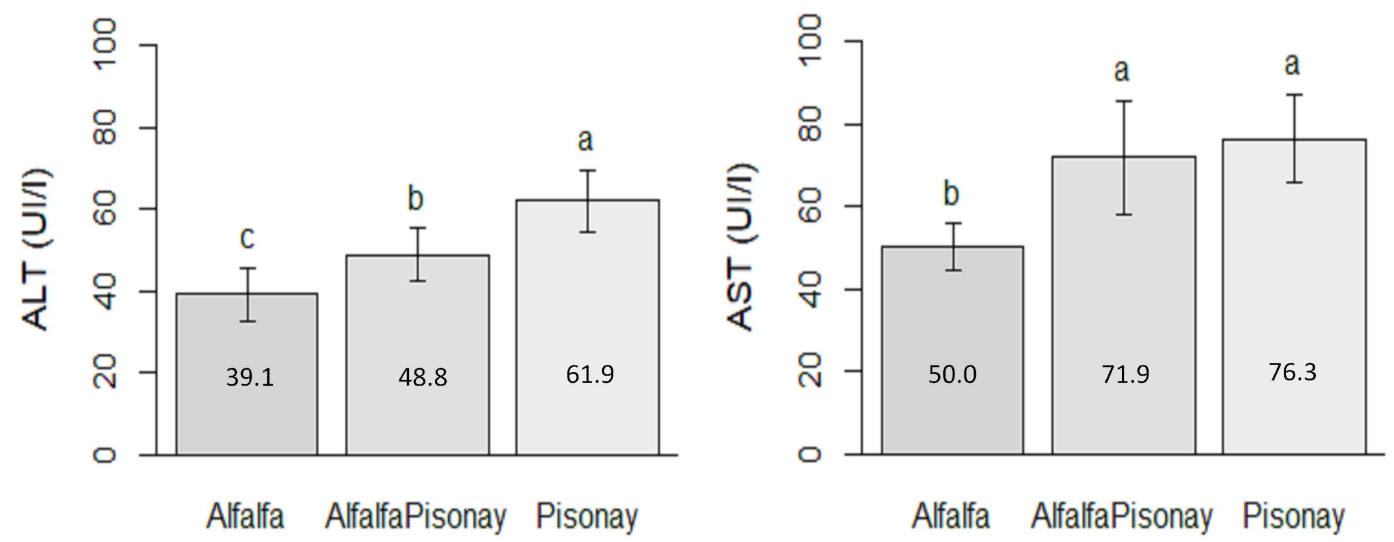

Figura 1. Niveles séricos de alanina aminotransferasa (ALT) y aspartato aminotransferasa (AST) en cuyes como respuesta a dietas de alfalfa (Medicago sativa) y pisonay (Erythrina $\mathrm{sp}$ )

significativos en ALT (24-39 UI/L) y AST (41$55 \mathrm{UI} / \mathrm{L}$ ), ni reacciones hepatotóxicas (Paredes et al., 2017). Estos resultados serían concordantes con los encontrados en esta investigación (Figura 1) mediante el consumo de alfalfa, pues dietas con $50 \%$ de pisonay o más serían más propensas a causar daño hepático.

Las tres dietas mostraron una presentación similar de congestión y dilatación vesicular (Cuadro 2); sin embargo, las dietas de pisonay en A50P50 y P100 conllevó a una mayor presencia de degeneración grasa $\mathrm{y}$ focos necróticos respecto a la dieta de solo alfalfa (Kumar, 1991). Las lesiones del híga- do (Figura 2) en los cuyes del presente estudio fueron similares a las producidas por plantas herbáceas, arbustos y arbóreas forrajeras. Avendaño (2011) menciona que alimentar cuyes con Carum carvi y Spilantehes oleraceae produce degeneración, necrosis y bilis con degeneración de estructura y color. Leucaena leucocephala procesada para alimentación de conejos ocasiona edema e hígado congestionado con manchas necróticas (Fayemi et al., 2011) y densa inflamación crónica del hígado (Adekojo et al., 2014). Por otro lado, la magnitud del incremento en la ALT, observada también en este estudio (Cuadro 2), refiere al grado de lesión de los hepatocitos (Thawley, 2017). 
Cuadro 2. Efecto de los alimentos sobre las lesiones del hígado de cuyes

\begin{tabular}{lccc}
\hline \multirow{2}{*}{ Lesiones } & \multicolumn{3}{c}{ Dietas } \\
\cline { 2 - 4 } & A100 & A50P50 & P100 \\
\hline Congestión & $37.5^{\mathrm{a}}$ & $87.5^{\mathrm{a}}$ & $75.0^{\mathrm{a}}$ \\
Degeneración grasa & $25.0^{\mathrm{a}}$ & $100.0^{\mathrm{b}}$ & $100.0^{\mathrm{b}}$ \\
Focos necróticos & $12.5^{\mathrm{a}}$ & $87.5^{\mathrm{b}}$ & $87.5^{\mathrm{b}}$ \\
Dilatación vesicular & $37.5^{\mathrm{a}}$ & $100.0^{\mathrm{a}}$ & $75.0^{\mathrm{a}}$ \\
Relación hígado/peso vivo (\%) & $4.1^{\mathrm{x}}$ & $3.8^{\mathrm{xy}}$ & $3.6^{\mathrm{y}}$ \\
\hline
\end{tabular}

a,b,c Superíndices diferentes dentro de filas denotan independencia; ${ }^{x, y}$ diferencia de medias A100: $100 \%$ Alfalfa (80 g), A50P50: 50\% Alfalfa (40 g) mas 50\% Pisonay (40 g) y P100: 100\% Pisonay (80 g)

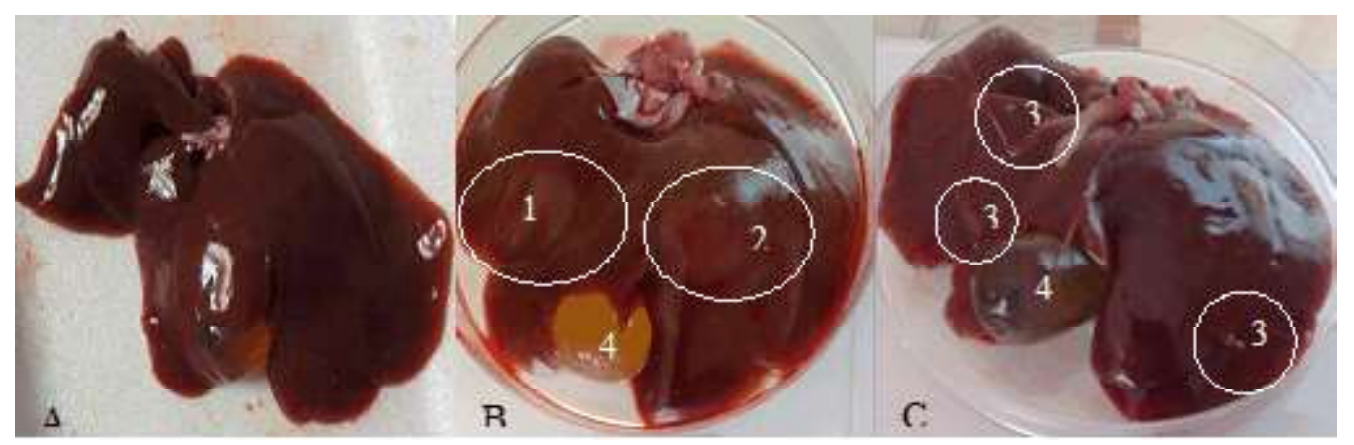

Figura 2. Hígado de cuyes. A. Hígado aparentemente normal de cuy alimentado con alfalfa. B. Hígado de cuy alimentado con alfalfa más pisonay. C. Hígado de cuy alimentado con pisonay. 1. Congestión. 2. Degeneración grasa. 3. Focos necróticos. 4. Dilatación vesicular

La relación hígado/peso vivo disminuyó al incrementarse el porcentaje de pisonay comparado con la dieta solo de alfalfa (Cuadro 2), lo cuál sería congruente con la observación en cuyes aparentemente sanos que tenían una relación entre 4.6 y $6.0 \%$ (Rosas, 2007; Stan et al., 2017). Del mismo modo, el peso del riñón, corazón, páncreas, proventrículo y bazo de gallos negros Nera estuvieron influenciados por dietas basadas en harina de hojas de M. oleifera, que ade- más afectaron con una respuesta elevada de la AST (Ojediran et al., 2017).

\section{Conclusiones}

Las hojas y peciolos del pisonay (Erythrina $\mathrm{sp}$ ) mostraron valores elevados de taninos y alcaloides que al ser suministrados como forraje fresco a partir de $50 \%$ en la dieta de cuyes; así mismo, aumentaron las 
concentraciones séricas de aminotransferasas, mayor presencia de patologías hepáticas y una disminución de la relación hígado/peso vivo.

\section{Literatura Citada}

1. Adekojo SA, Adama TZ, Aremu A, Ijaiya AT, Owoleke OE, Ibrahim A. 2014. Effects of dietary inclusion of differently processed Leucaena leucocephala leaf meal on carcass characteristics of rabbits (Oryctolagus cunniculus). Int J Food Sci Nutr Engineering 4: 118-127. doi: 10.5923/ j.food.20140405.02

2. Alikwe PCN, Omotosho MS. 2013. An evaluation of the proximate and phytochemical composition of Moringa oleifera leaf meal as potential feedstuff for non-ruminant livestock. Agrosearch 13: 17-27. doi: 10.4314/agrosh.v13i1.2

3. Alvear CM, Melo W, Apráez JE, Gálvez A, Insuasty EG. 2013. Especies arbóreas y arbustivas con potencial silvopastoril en la zona de bosque muy seco tropical del norte de Nariño y sur del Cauca. Agroforestería Neotropical 3: 37-46.

4. Apráez JE, Gómez TC, Calpa JS. 2013. Comportamiento productivo de cuyes (Cavia porcellus) bajo arreglos silvopastoriles en clima medio del departamento de Nariño, Colombia. Rev Inv Pec 2: 41-48.

5. Apráez E, Gálvez A, Navia J. 2017. Evaluación nutricional de arbóreas y arbustivas de bosque muy seco tropical (bms-T) en producción bovina. Rev Cienc Agron 34: 98-107. doi: 10.22267/ rcia.173401.66

6. Araújo-Júnior JX, Oliveira MSG, Aquino PGV, Alexandre-Moreira MS, Sant'Ana AEG. 2012. A phytochemical and ethnopharmacological. Review of the genus Erythrina. In: Venketeshwer Rao (ed). Phytochemicals - a global perspective of their role in nutrition and health. Rijeka: China: InTech. p 327-352.
7. [AOAC] Association of Official Analytical Chemists. 1990. Official methods for analysis of the AOAC. $15^{\text {th }}$ ed. Arlington, Virginia, EEUU: AOAC. $771 \mathrm{p}$.

8. Astaiza-Martínez JM, BenavidesMelo CJ, Chaves-Velásquez CA, Arciniegas-Rivera AM, Quiroz-Moran LH. 2013. Estandarización de la técnica de necropsia en cuyes (Cavia porcellus) en la Universidad de Nariño. Rev Inv Pec 2: 77-83.

9. Avendaño MM. 2011. Determinación de alteraciones orgánicas causadas por plantas tóxicas, alcaravea (Carum carvi) y botoncillo (Spilantehes oleraceae) en cobayos. Tesis de Médico Veterinario Zootecnista. Loja: Univ. Nacional de Loja. $72 \mathrm{p}$.

10. Barry TN, McNabb WC. 1999. The effect of condensed tannins in temperate forages on animal nutrition and productivity. In: Brooker JD (ed). Tannins in livestock and human nutrition. Camberra: Center for International Agricultural. p 30-35.

11. Bhat TK, Kannan A, Birbal S, Sharma OP. 2013. Value addition of feed and fodder by alleviating the antinutritional effects of tannins. Agric Res 2: 189-206. doi: 10.1007/s40003-013-0066-6

12. Bohada CM, Ospina LA, Vargas JE. 2017. Identificación y caracterización de especies vegetales con potencial forrajero en trópico alto de la cuenca del rio Tapias. Livestock Res Rural Develop 29(5). [Internet]. Disponible en: http:// www.lrrd.org/lrrd29/5/karm29100.html

13. Brown D, Ng'ambi JW, Norris D, Mbajiorgu FE. 2016. Blood profiles of indigenous Pedi goats fed varying levels of Vachellia karroo leaf meal in Setaria verticillata hay-based diet. S Afr J Anim Sci 46: 432-440. doi: 10.4314/sajas.v46i4.11

14. Cárdenas LA, Bautista JL, Zegarra JL, Ramos R. 2013. Degradabilidad ruminal de la fibra del follaje pisonay (Erythrina sp). Rev Complutense Cienc Vet 7: 42-49. doi: 10.5209/rev_RCCV.2013.v7.n1.41687 
15. Cárdenas LA, Bautista JL, Zegarra JL, Ramos R, Gomez OE, Barreto, J.S. 2016a. Degradabilidad in situ de la materia seca y proteína cruda de las hojas y peciolo del pisonay (Erythrina falcata). Rev Inv Vet Perú 27: 39-44. doi: 10.15381/rivep.v27i1.11461

16. Cárdenas LA, Sánchez CZ, Ramírez Y, Ramos R. 2016b. Uso del pisonay (Erythrina sp) en la alimentación de cuyes. En: Simposio Nacional Avances y Perspectivas en la Producción de Cuyes. Lima, Perú. UNALM.

17. Cheema UB, Sultan JI, Javaid A, Akhtar P, Shahid M. 2011. Chemical composition, mineral profile and in situ digestion kinetics of fodder leaves of four native trees. Pak J Bot 43: 397-404. doi: 10.4314/sajas.v46i4.11

18. Choque H, Huaita A, Cárdenas LA, Ramos R. 2018. Efecto de la edad de rebrote en la degradación ruminal del pisonay (Erythrina sp) en el valle interandino de Abancay. J High Andean Res 20: 189-202. doi: 10.18271/ ria. 2018.363

19. Coblentz WK, Grabber JH. 2013. In situ protein degradation of alfalfa and birdsfoot trefoil hays and silages as influenced by condensed tannin concentration. J Dairy Sci 96: 3120-3137. doi: 10.3168/jds.2012-6098

20. Fayemi P, Onwuka CF, Isah OA, Jegede AV, Arigbede OM, Muchenje V. 2011. Effects of mimosine and tannin toxicity on rabbits fed processed Leucaena leucocephala (Lam) De Wit. Leaves. Afr J Agr Res 6: 4081-4085. doi: 10.5897/AJAR11.327

21. Galindo J, González N, Marrero Y, Sosa A, Ruiz T, Febles G, Torres V, Aldana AI, Achang G, Moreira O, Sarduy L, Noda AC. 2014. Efecto del follaje de plantas tropicales en el control de la producción de metano y la población de protozoos ruminales in vitro. Cuban J Agr Sci 48: 359-364.

22. García DE, Medina MG 2006. Composición química, metabolitos secundarios, valor nutritivo y aceptabilidad rela- tiva de diez árboles forrajeros. Zootecnia Trop 24: 233-250.

23. García DE, Medina MG, Clavero T, Cova LJ, Domínguez C, Baldizán A. 2008. Caracterización nutritiva del follaje de seis especies forrajeras con énfasis en sus perfiles polifenólicos. Rev Cient Fac Cien V 18: 188-196.

24. García DE, Medina MG, Moratinos P, Cova LJ, Torres A, Santos O, Perdomo D. 2009. Caracterización químiconutricional de forrajes leguminosos y de otras familias botánicas empleando análisis descriptivo y multivariado. Avances Invest Agropec 13: 25-39.

25. Guevara J, Díaz P, Bravo N, Vera M, Crisóstomo O, Barbachán H, Huamán D. 2013. Uso de harina de pajuro (Erythrina edulis) como suplemento en la alimentación de cuyes-Lima. Rev Per Quím Ing Quím 16: 21-28.

26. Jurado-Gámez HA, Cabrera EJ, Salazar JA. 2016. Comparación de dos tipos de sacrificio y diferentes tiempos de maduración sobre variables fisicoquímicas y microbiológicas de la carne de cuy (Cavia porcellus). Rev Med Vet Zoot 63: 201-217. doi: 10.15446/ rfmvz.v63n3.62741

27. Kitagaki M, Yamaguchi M, Nakamura M, Sakurada K, Suwa T, Sasa H. 2005. Age-related changes in haematology and serum chemistry of Weiser-Maples guinea pigs (Cavia porcellus). Lab Anim 39: 321-330. doi: 10.1258/0023677054307042

28. Kongmanila D, Bertilsson J, Ledin I, Wredle E. 2012. Utilisation of some Erythrina species and biomass production of Erythrina variegate. Livestock Res Rural Develop 24(8). [Internet]. Disponible en: http:// www.lrrd.org/lrrd24/8/daov24137.htm

29. Kumar R. 1991. Anti-nutritional factors: the potential risks of toxicity and methods to alleviate them. In: Speedy A, Pugliese JL (eds). Legume trees and other fodder trees as protein sources for livestock. Proc FAO Expert Consultation MARDI. Kuala Lumpur, Malaysia. 
30. Lawrence YA, Steiner JM 2017. Laboratory evaluation of the liver. Vet Clin N Am-Small 47: 539-553. doi: 10.1016/j.cvsm.2016.11.005

31. Mashamaite L, Ng'ambi JW, Norris $D, N d l o v u L R$ and Mbajiorgu CA. 2009. Relationship between tannin contents and short-term biological responses in male rabbits supplemented with leaves of different acacia tree species grown in Limpopo province of South Africa. Livestock Res Rural Develop 21(7). [Internet]. Disponible en: http://www.1rrd.org/1rrd2 1/7/ mash21109.htm

32. Meza GA, Sánchez AR, Meza MA, Meza CJ, Franco NG, Avellaneda JH, Estupiñán KA, et al. 2012. Digestibilidad in vivo de forrajeras arbustivas tropicales para la alimentación de cuyes (Cavia porcellus Linnaeus), en el litoral ecuatoriano. Vet Zootec 6: 8-16.

33. Meza GA, Loor NJ, Sánchez AR, Avellaneda JH, Meza CJ, Vera DF, Cabanilla MG, et al. 2014a. Inclusión de harinas de follajes arbóreos y arbustivos tropicales (Morus alba, Erythrina poeppigiana, Tithonia diversifolia e Hibiscus rosa-sinensis) en la alimentación de cuyes (Cavia porcellus Linnaeus). Rev Fac Med Vet Zoot 61: 258-269. doi: 10.15446/ rfmvz.v61n3.46874

34. Meza GA, Cabrera RP, Morán JJ, Meza FF, Cabrera CA, Meza JC, Meza JS, et al. 2014b. Mejora de engorde de cuyes (Cavia porcellus L.) a base de gramíneas y forrajeras arbustivas tropicales en la zona de Quevedo, Ecuador. Idesia 32: 75-80.

35. Milián JC, Iglesias O, Valdés H. 2017. Caracterización fitoquímica de Samanea Saman Jacq Merr (algarrobo). Rev Cub Cienc Forestales 5: 49-61.

36. Mohamed KE, Salih AM, Zomrawi WB, Hamza MM, Elamin KM, Dousa BM. 2015. The effect of drying methods on chemical composition and digestibility of Leucaena Leucocephala leaves. Global J Anim Sci Res 3: 419-422.

37. Ogunbosoye DO, Tona GO, Otukoya FK. 2015. Evaluation of the nutritive value of selected browse plant species in the southern guinea savannah of Nigeria for feeding to ruminant animals. Brit J Appl Sci Technol 7: 386-395. doi: 10.9734/BJAST/2015/14051

38. Ojeda A, Barroso JA, Obispo N, Gil JL, Cegarra R. 2012. Composición química, producción de gas in vitro y astringencia en el follaje de Samanea saman (Jacq.) Merrill. Pastos y Forrajes 35: 205-218.

39. Ojediran T, Oyebode O, Amao O, Shittu D, Odedoyin O. 2017. Serum biochemistry, organ weight, carcass characteristics, organoleptic properties and villi morphometry of Nera Black Cocks fed varying levels of Moringa oleifera leaf meal. Anim Sci Biotechnol 50: $16-23$.

40. Paredes-Lopez, D, Robles-Huaynate $R$, Córdova-Chumbes O, De la CruzPaucar E. 2017. Efecto de la harina de hojas de Erythrina sp sobre el perfil bioquímico, parámetros biológicos e histopatología del hígado de Cavia porcellus. Scientia Agropec 8: 297-304. doi: 10.17268/sci.agropecu.2017.04.01

41. Pedraza RM, Martínez SJ, Estévez $J A$, Guevara GF, Guevara $R E$, Curbelo L. 2007. Valor nutritivo para rumiantes del follaje de árboles y arbustos tropicales. Rev Prod Anim 19: 5-12.

42. Pilny AA. 2008. Clinical hematology of rodent species. Vet Clin North Am Exotic Anim Pract 11: 523-533. doi: 10.1016/ j.cvex.2008.04.001

43. Pino S, Prieto S, Pérez ME, Molina J. 2004. Género Erythrina: fuente de metabolitos secundarios con actividad biológica. Acta Farm Bonaerense 23: 252-258.

44. Reddy DV, Elanchezhian N. 2008. Evaluation of tropical tree leaves as ruminant feedstuff based on cell 
contents, cell wall fractions and polyphenolic compounds. Livestock Res Rural Develop 20(5). [Internet]. Disponible en: http://www.1rrd.org/1rrd20/5/ redd20077.htm

45. Rodríguez Y, Chongo B, La OO, Oramas A, Scull I, Achang, G. 2004. Tamizaje fitoquímico de Erythrina mysorensis y determinación de su potencial nutritivo mediante la técnica de producción de gas in vitro. Estudio preliminar. Cuban J Agr Sci 38: 161-166.

46. Rosas C. 2007. Aspectos anatómicos, histológicos, histoquímicos y estereológicos del hígado de cobayo (Cavia porcellus). Tesis de Tecnólogo Médico. Temuco: Univ. de la Frontera. 68 p.

47. Ruíz-RodríguezJ. 2013. Aproximación al análisis de bioquímica sanguínea y uroanálisis en animales silvestres y especies no convencionales. Mem Conf Interna Med Aprovech Fauna Silv Exót Conv 9: 58-67.

48. Sánchez A, Zambrano D, Torres E, Meza $G$ 2012. Forrajeras tropicales y banano maduro (Musa paradisiaca) en el engorde de cuyes (Cavia porcellus L.) en el cantón Quevedo. Actas Iberoam Conservación Animal 2: 287-290.

49. Santacoloma LE, Granados JE. 2012. Interrelación entre el contenido de metabolitos secundarios de las especies Gliricidia sepium y Tithonia diversifolia y algunas propiedades físicoquímicas del suelo. Rev Invest Agraria Amb 3: 53-62.

50. Santos M, Savón L, Lon-Wo E, Gutiérrez O, Herrera M. 2014. Inclusión de harina de hojas de Morus alba: su efecto en la retención aparente de nutrientes, comportamiento productivo y calidad de la canal de pollos cuello desnudo. Cuban J Agr Sci 48: 259-264.

51. Stan FG, Martonos C, Dezdrobitu C, Damian A, Gudea A. 2017. Detailed morphological description of the liver and hepatic ligaments in the guinea pig $(\mathrm{Ca}$ via porcellus). Sci Works Series C Vet Med 63: 35-42.

52. Thawley V. 2017. Acute liver injury and failure. Vet Clin N Am-Small 47: 617630. doi: 10.1016/j.cvsm.2016.11.010.

53. Ticona MM. 2011. Actividad enzimática de las transaminasas glutámico oxalacético, glutámico pirúvico y fosfatasa ácida en el suero sanguíneo del cuy. Tesis de Médico Veterinario Zootecnista. Puno: Univ. Nacional del Altiplano. $54 \mathrm{p}$.

54. Tijani LA, Akanji AM, Agbalaya K, Onigemo M. 2016. Comparative effects of graded levels of Moringa leaf meal on haematological and serum biochemical profile of broiler chickens. J Agr Sci 11: 137-146. doi: 10.4038/ jas.v11i3.8167

55. Velásquez AM. 2004. Extracción de taninos presentes en el banano verde. Rev Lasallista Investig 1: 17-22.

56. Yisehak K, Janssens GPJ. 2013. Evaluation of nutritive value of leaves of tropical tanniferous trees and shrubs. Livestock Res Rural Develop 25(2). [Internet]. Disponible en: https:// www.1rrd.cipav.org.co/1rrd25/2/ yise25028.htm

57. Zhu J, Filippich LJ, Alsalami MT. 1992. Tannic acid intoxication in sheep and mice. Res Vet Sci 53: 280-292. doi: 10.1016/0034-5288(92)90128-O 